\title{
THIN FILM CRYSTALLINITY AND SUBSTRATE MATERIALS IN ATOMIC GRAPHOEPITAXY $\mathrm{OF} \mathrm{YBa}_{2} \mathrm{Cu}_{3} \mathrm{O}_{x}$
}

\author{
S. Miyazawa, M. Mukaida and M. Sasaura \\ NTT System Electronics Laboratories \\ 3-1, Morinosato Wakamiya, Atsugi-shi, Kanagawa 243-01, Japan
}

\begin{abstract}
The complexity of $a-/ c$-axis oriented growth of $\mathrm{YBa}_{2} \mathrm{Cu}_{3} \mathrm{O}_{x}$ thin films is reviewed from the viewpoint of controlling $a$-axis preferred thin film growth. Perfectly $c$-axis in-plane-aligned $a$-axis oriented $\mathrm{YBa}_{2} \mathrm{Cu}_{3} \mathrm{O}_{x}$ thin films, or "pure" $a$-axis oriented films, can be grown on the (100) face of tetragonal $\mathrm{K}_{2} \mathrm{NiF}_{4}$-type substrates by template growth process. A new growth mechanism called atomic graphoepitaxy is presented as a growth model. Transmission electron microscopy reveals that the films on a (100) $\mathrm{SrLaGaO}_{4}$ substrate consist of domains surrounded by anti-phase and stacking-fault boundaries. This domain formation can be well explained by substrate surface irregularities inherent in the $\mathrm{SrLaGaO}_{4}$ crystal. The formation of defects in microstructures in "pure" $a$-axis oriented $\mathrm{YBa}_{2} \mathrm{Cu}_{3} \mathrm{O}_{x}$ thin films is also modeled based on our atomic graphoepitaxial growth model.
\end{abstract}

PACS numbers: $74.76 . \mathrm{Bz}, 74.80 .-\mathrm{g}, 68.35 .-\mathrm{p}$

\section{Introduction}

Since the discovery of $\mathrm{YBa}_{2} \mathrm{Cu}_{3} \mathrm{O}_{x}$ (YBCO), thin film growth of $\mathrm{YBCO}$ with a $T_{\mathrm{c}}$ of $90 \mathrm{~K}$ has been investigated to realize trilayered superconductor/insulator/ superconductor (SIS) Josephson tunnel junctions. Ilowever, no clear Josephson tunneling characteristics have been observed to date. The most important issue for making SIS trilayer structures is the growth of thin films in which each layer has an atomically smooth surface. Since film surface morphology strongly depends on growth modes or the initial growth stages of thin films, substrate materials play an important role from the viewpoint of epitaxy. Studies on the causal relationship between substrate surface and the initial growth stages of YBCO thin films are common to establish the growth of an insulating barrier layer in Josephson tunnel SIS structures.

$c$-axis oriented YBCO thin films grow in Stranski-Krastanov and/or VolmerWeber modes when using conventional physical vapor deposition (PVD) techniques, even if lattice-matched substrates with perovskite-related structures are used. The essential parameters that dominate the growth modes are the degree of "lattice-matching" and the degrce of "supersaturation" [1,2]. The degree of 
"supersaturation" is influenced by the deposition rate and oxygen partial pressure in PVD techniques. In fact, atomically smooth $c$-axis oriented films were grown when the deposition rate, usually about $0.1 \mu \mathrm{m} / \mathrm{s}$, was decreased by an order of magnitude in MBE technique [3]. The grown $c$-axis films, however, easily crystallize with spiral growth associated with screw dislocations [4], which are due to a large Burger vector of YBCO. Thus, spiral growth is the biggest obstacle in preparing $c$-axis oriented films with atomically smooth surfaces.

On the other hand, $a$-axis oriented films are favorable for realizing trilayered SIS structures because their coherence length $\xi_{a}$ is longer than that along the $c$-axis direction, $\xi_{c}$. Many attempts to grow $a$-axis oriented YBCO thin films have been reported so far. For example, Eom et al. [5] first succeeded in growing $a$-axis oriented YBCO films using an $a$-axis oriented, non-superconducting $\mathrm{PrBa}_{2} \mathrm{Cu}_{3} \mathrm{O}_{\mathrm{x}}$ (PrBCO) buffer layer. These $a$-axis preferred YBCO thin films grow easily when growth temperatures are lower than the usual $c$-axis oriented film growth temperatures by $\approx 100^{\circ} \mathrm{C}$. However, most of the $a$-axis oriented films on perovskite-related substrates, such as $\mathrm{SrTiO}_{3}, \mathrm{LaAlO}_{3}$ and $\mathrm{NdGaO}_{3}$, showed an extremely low $T_{\mathrm{c}}$ of $\approx 20 \mathrm{~K}$, as compared with $\approx 90 \mathrm{~K}$ for $c$-axis oriented films. Most of the films consisted of $c$-axis in-plane orthogonally aligned grains, that is, $90^{\circ}$-domains. Why the $T_{\mathrm{c}}$ 's of most $a$-axis films are low is not clear, but one way to obtain $a$-axis oriented films with a $T_{\mathrm{c}}$ of $\approx 90 \mathrm{~K}$ may be to suppress the $90^{\circ}$-domains in the films, resulting in perfectly $c$-axis in-plane-aligned films.

This paper briefly describes recent understandings of the growth of $a$-axis oriented YBCO thin films with a $T_{\mathrm{c}}$ of $\approx 90 \mathrm{~K}$. It focuses on a new epitaxial growth model called "atomic graphoepitaxy" for growing $c$-axis in-plane aligned, $a$-axis oriented YBCO thin films, that is, "pure" $a$-axis oriented films, on $\mathrm{K}_{2} \mathrm{NiF}_{4}$-type substrates, and discusses the formation model of in-plane lattice defects in the films.

\section{Control of $a-/ c$-axes oriented growth of YBCO thin films}

Figure 1 summarizes the dependence of the preferred orientation of the YBCO thin films on growth temperature $\left(p\left(\mathrm{O}_{2}\right)=400 \mathrm{~m} T r\right)$, for films grown on different substrate materials with perovskite-related structures [6]. The film deposition was carried out by an $\mathrm{ArF}$ excimer laser ablation technique. In the figure, lattice constants, excluding those of $\mathrm{SrTiO}_{3}$ and $\mathrm{MgO}$, are of pseudo-cubic system and are plotted with regard to thermal expansivity. We can see that the increase in growth temperature results in a change from $a$-axis-dominant to $c$-axis-dominant orientation and the transition temperature from $a$-axis to $c$-axis orientation depends on the substrate used, that is, the smaller the lattice mismatch, the higher the transition temperature. However, the $T_{\mathrm{c}}$ of $a$-axis oriented films grown in this way was as low as $20 \mathrm{~K}$, while the $T_{\mathrm{c}}$ of the $c$-axis oriented films was more than $85 \mathrm{~K}$. Careful observations of the $a$-axis oriented films by transmission electron microscopy (TEM) and X-ray $\phi$-scanned diffraction clearly indicated orthogonally $c$-axis in-plane-aligned grains in the films, called $90^{\circ}$-domains.

Detailed investigations on the preferred orientation depending on growth conditions showed that a lower growth temperature and higher oxygen partial pressure resulted in preferentially $a$-axis oriented films. This guideline was also 


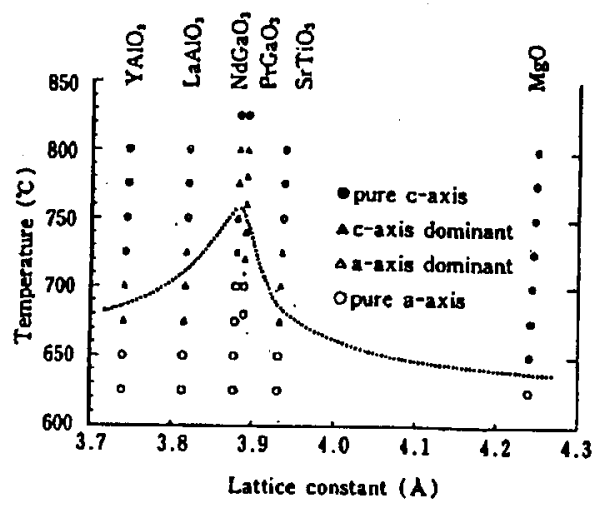

Fig. 1. Dependence of the preferred film orientation on growth temperature for different perovskite-related substrates.

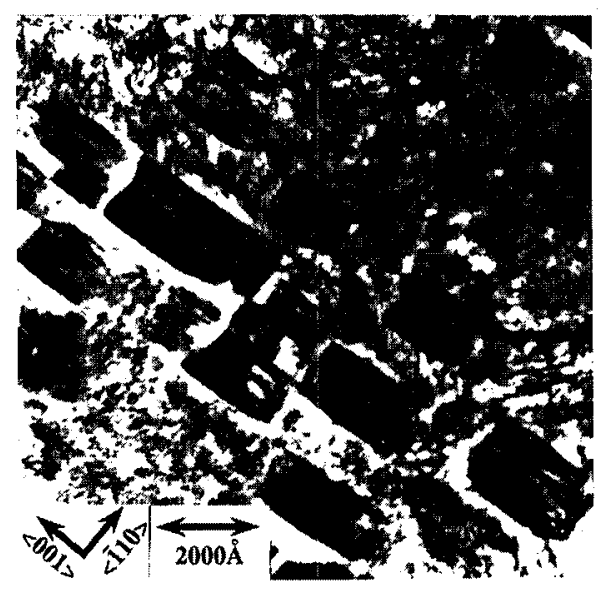

Fig. 2. Planar-view TEM image on a (110) $\mathrm{NdGaO}_{3}$ substrate, where almost all $a$-axis oriented grains are aligned along the [001] on the substrate surface.

confirmed in $a$-axis growth by reactive co-evaporation (MBE) method [7]. We investigated $a$-axis oriented grains in $c$-axis dominated film grown under higher growth temperatures and higher oxygen partial pressure on a (110) $\mathrm{NdGaO}_{3}$ substrate [8]. Figure 2 shows a planar TEM image of thin film grown at $800^{\circ} \mathrm{C}$, in which many grains in the film matrix are clearly aligned in one direction parallel to the in-plane $\langle 001\rangle$ of the substrate surface. To verify the orientation relationship between the grains and the substrate, X-ray $\phi$-scan diffraction using a (102) peak of the film was carried out. Figure 3 shows the $\phi$-scanned diffraction pattern, where sharp peaks are located only at $\phi=n \pi$ ( $n$ : integer), indicating that the $c$-axis of the grains is ordered in-plane and aligned in the direction of $\langle-110\rangle$ of the (110) $\mathrm{NdGaO}_{3}$ substrate. The cell constant along the $\langle-110\rangle$ is longer than that along the $\langle 001\rangle$ at $800^{\circ} \mathrm{C}$ because of the pseudo-tetragonality of $\mathrm{NdGaO}_{4}[9,10]$. This 


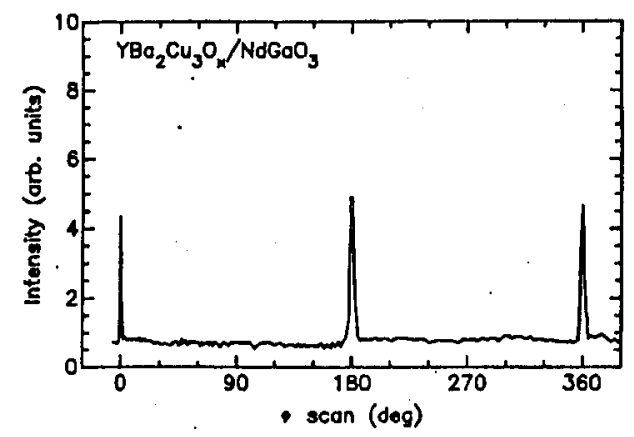

Fig. 3. X-ray $\phi$-scanned pattern of a film on a (110) $\mathrm{NdGaO}_{3}$ substrate, grown under high growth temperature and high partial oxygen pressure.

geometrical analysis led us.to conclude that the tetragonality of the substrate surface lattice should be the key for growing $c$-axis in-plane aligned, $a$-axis oriented, or "pure" $a$-axis oriented YBCO thin films.

\section{Growth of "pure" a-axis YBCO thin films}

Based on the supposition above, tetragonal substrates having lattice constants matched with both $b$ - and $c$-axes lengths of YBCO should be ideal for growing "pure" $a$-axis oriented YBCO thin films from the epitaxy point of view. We searched for materials that consist of oxygen octahedra with a tetragonal structure, such as crystals with the tetragonal $\mathrm{K}_{2} \mathrm{NiF}_{4}$ structure.

Hontsu et al. [11] succeeded in growing the "pure" a-axis YBCO thin films using the (100) face of tetragonal $\mathrm{SrLaGaO}_{4}$ substrates with a buffer layer of PrBCO. Independently, we also succeeded in growing "pure" $a$-axis oriented films directly on (100) $\mathrm{SrLaGaO}_{4}$ substrates without any buffer layers [12]. Lattice constants of $\mathrm{SrLaGaO}_{4}$ are $3.843 \AA$ and $12.61 \AA$ for $a_{\mathrm{T}^{-}}$and $c_{\mathrm{T}}$-axes, respectively. Instead of the buffer layer deposition, we used the template technique, in which the initial deposition was carried out at about $640^{\circ} \mathrm{C}$ for a few minutes (equivalent to $\approx 230 \AA$ in thickness) and the substrate temperature was increased to about

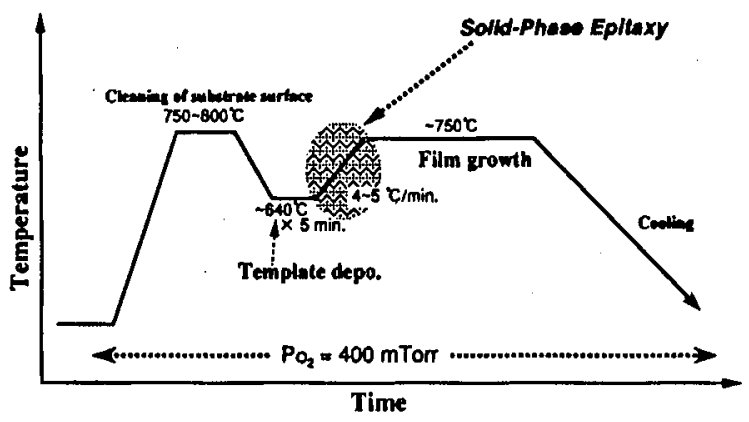

Fig. 4. Growth program of "pure" $a$-axis oriented YBCO thin films. 


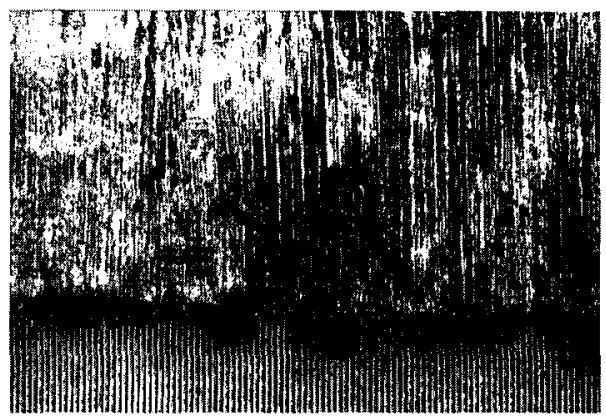

Fig. 5. Cross-sectional TEM image at the interface between $\mathrm{SrLaGaO}_{4}$ and $\mathrm{YBCO}$ thin film, viewed from the [010] axis.

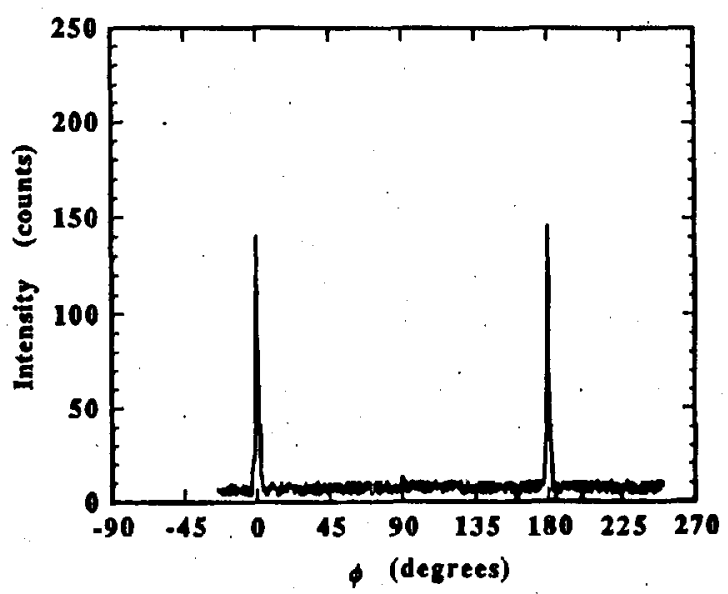

Fig. 6. X-ray $\phi$-scanned diffraction pattern with (102) reflection of, YBCO thin film on (100) $\mathrm{SrLaGaO}_{4}$.

$750^{\circ} \mathrm{C}$ to ensure sufficient absorption of oxygen in to the films. This growth program is illustrated in Fig. 4. Typical as-grown film thickness was about $2700 \AA$. This template technique plays an important role, in which a kind of a solid-phase epitaxy (SPE) takes place, as will be discussed later.

Figure 5 shows a TEM image at the interface between $\mathrm{SrLaGaO}_{4}$ substrate and YBCO film viewed from the $b$-axis of the film. Notice that there is no distinct interfacial layer such as disordered pseudo-cubic phase $[13,14]$. Detailed X-ray diffraction and RHEED experiments showed that the film is a perfectly $c$-axis in-plane-aligned, $a$-axis oriented film [12]. For example, Fig. 6 shows an X-ray $\phi$-scanned diffraction pattern with (102) reflection, where peaks appear at each $180^{\circ}$ indicating it free of $90^{\circ}$-domains.

Figure 7 depicts temperature dependence of resistivity along both in-plane $b$ - and $c$-directions in as-grown state. $T_{\mathrm{c}}$ was measured to be $\approx 90 \mathrm{~K}$ with $\Delta T_{\mathrm{c}}$ of $\approx 1 \mathrm{~K}$ with metallic behaviors. The ratio of $\rho_{c} / \rho_{b}$ at $100 \mathrm{~K}$ was about 22 [15]. 


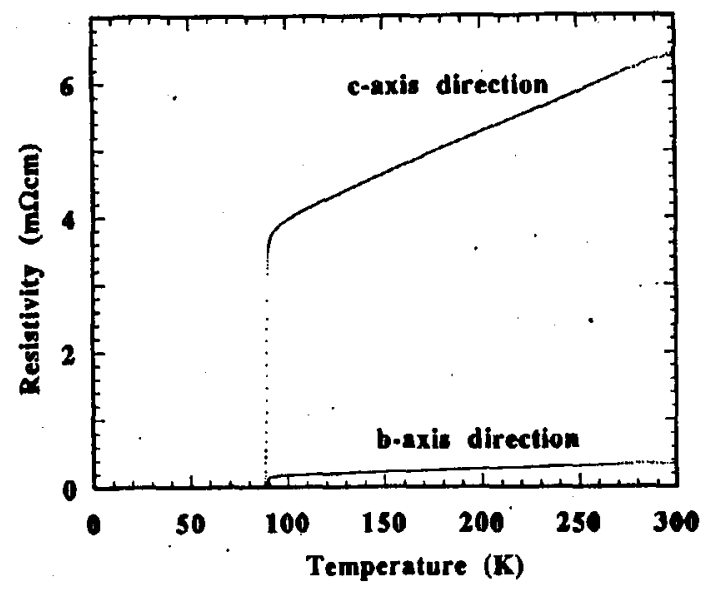

Fig. 7. Temperature dependence of resistivity along in-plane $b$ - and $c$-axes of the "pure" $a$-axis oriented YBCO thin film.

To confirm "pure" a-axis YBCO thin film growth on tetragonal (100) $\mathrm{K}_{2} \mathrm{NiF}_{4}$ substrates, a tetragonal $(100)$ oriented $\mathrm{Nd}_{2} \mathrm{CuO}_{4}$ crystal $\left(a_{\mathrm{T}}=3.94 \AA, c_{\mathrm{T}}=\right.$ $12.15 \AA$ ) was used as a substrate, and YBCO thin film was grown according to the procedure illustrated in Fig. 4. X-ray diffractions and RHEED observations proved that the film was also perfectly $c$-axis in-plane aligned, $a$-axis oriented one. For $\mathrm{Nd}_{2} \mathrm{CuO}_{4}$, lattice mismatches at $\approx 700^{\circ} \mathrm{C}$ of growth temperature for $b$ - and $c$-axis directions are estimated to be respectively $+1.83 \%$ and $+3.45 \%$. $T_{\mathrm{c}}$ 's were verified to be $\approx 90 \mathrm{~K}$ for both $b$ - and $c$-axis directions. Additionally, a (100) $\mathrm{Ce}: \mathrm{Nd}_{2} \mathrm{CuO}_{4}$ substrate also resulted in "pure" $a$-axis oriented $\mathrm{YBCO}$ thin films. $\mathrm{SrPrGaO}_{4}$ single crystal, a new type of $\mathrm{K}_{2} \mathrm{NiF}_{4}$ compound, were grown in house [16], as shown in Fig. 8. Lattice constants $a_{\mathrm{T}}$ and $c_{\mathrm{T}}$ are respectively $3.823 \AA$ and $12.578 \AA$, and the lattice-mismatches at $700^{\circ} \mathrm{C}$ are $-1.18 \%$ and $+6.40 \%$ for $a$ and $c$-axes, respectively. Tentative growth of YBCO thin films on a (100) $\mathrm{SrPrGaO}_{4}$ substrate proved that the films are "pure" $a$-axis oriented YBCO thin films, but crystalline quality was worse, to some extent, compared with that on $\mathrm{SrLaGaO}_{4}(\delta a=-0.66 \%$ and $\delta c=+7.72 \%$ at $700^{\circ} \mathrm{C}$ ). The in-plane orientation relationship between thus grown

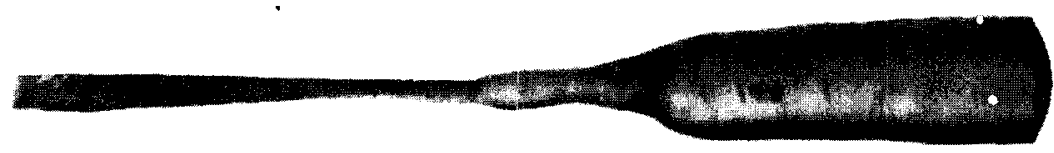

Fig. 8. As-grown single crystal of a new $\mathrm{K}_{2} \mathrm{NiF}_{4}$ crystal, $\mathrm{SrPrGaO}_{4}$.

all of "pure" $a$-axis YBCO film and the tetragonal $\mathrm{K}_{2} \mathrm{NiF}_{4}$-type substrates was [010]YBCO $\|[100]$ sub. and [001] YBCO $\|[001]$ sub.

Using the "pure" $a$-axis YBCO thin films, we could carefully examine the resistivity broadening phenomena along both $b$ - and $c$-axes of the film [15]. 


\section{Atomic graphoepitaxial model}

A new growth model called "atomic graphoepitaxy" [17] for the "pure" a-axis oriented YBCO thin film on $\mathrm{K}_{2} \mathrm{NiF}_{4}$ substrates was hypothesized by analogy to graphoepitaxy. Smith and Flanders' paper on graphoepitaxy [18] (In Greek, grapho means write or incise, epi does upon, and taxy does arrangement or ordering.) presented the growth of $\mathrm{Si}$ thin film with preferred orientation on $\mathrm{SiO}_{2}$ glass substrates. They investigated nucleation of $\mathrm{Si}$ on artificial corrugations or grooves made by conventional photolithography on glass substrates. Surface grooves act as nucleation sites of oriented Si nuclei deposited from vapor phase. Smith said that ultimate line-and-space corrugation is crystal lattices [19]. This encouraged our atomic graphoepitaxy model which is demonstrated in Fig. 9 [17].

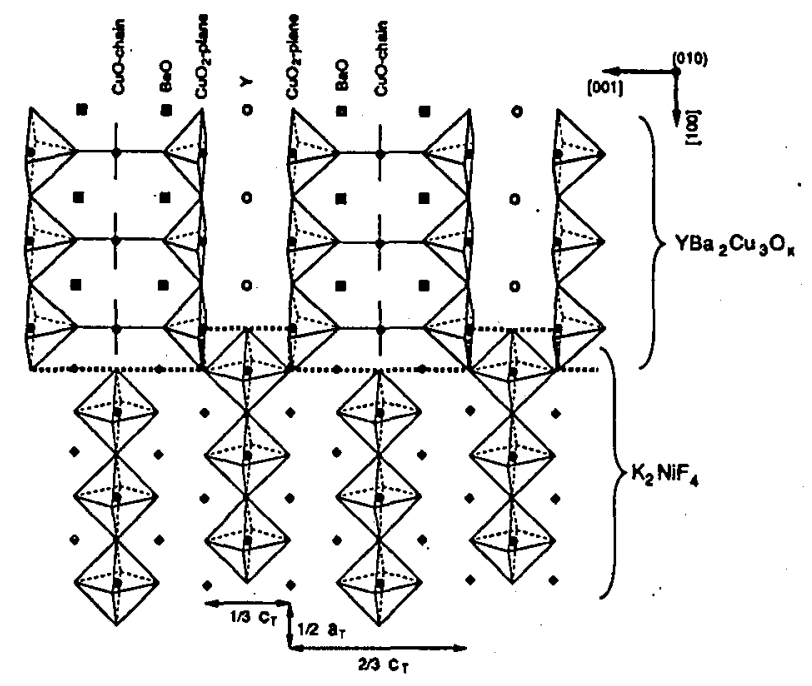

Fig. 9. Idealized arrangement of atomic graphoepitaxial interface between YBCO and $\mathrm{K}_{2} \mathrm{NiF}_{4}$ substrate, projected on $\{010\}$.

In Fig. 9 we illustrated the surface atomic arrangement of tetragonal $\{100\}$ face of $\mathrm{K}_{2} \mathrm{NiF}_{4}$-type crystal. The lower part shows a $\langle 010\rangle$ projection of the crystal, where the (100) surface consists of oxygen octahedra - $\left[\mathrm{GaO}_{6}\right]$ - forming a rectangular corrugated relief with $2 c_{\mathrm{T}} / 3$-wide grooves and $1 c_{\mathrm{T}} / 3$-wide ridges, as shown by dotted lines. This atomic corrugation runs along the [010] direction and the rectangular grooves or ridges are perpendicular to the [001] direction. A preliminary trial for observing atomic surface corrugations by atomic force microscopy (AFM) proved that the surface was roughly periodic corrugation with multiples of $\approx 4 \AA$ concavity/convexity [2]. The upper part also shows a $\langle 010\rangle$ projection of the YBCO crystal. The $\{010\}$ face of YBCO also consists of oxygen-deficient octahedra centered $\mathrm{Cu}$, forming rectangular saw-toothed corrugation with $2 c_{\mathrm{T}} / 3$-wide ridges and $1 c_{\mathrm{T}} / 3$-wide grooves running along the [010] direction. This feature is quite similar to that of the (010) face of the $\mathrm{K}_{2} \mathrm{NiF}_{4}$ structure. It can be easily imaged that both rectangular saw-tooth relieves can fit tightly into each other 


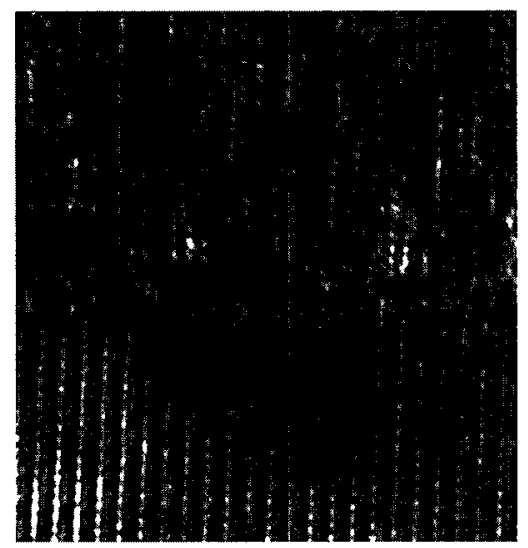

Fig. 10. Enlarged TEM image at the interface between "pure" $a$-axis YBCO thin film and $\mathrm{SrLaGaO}_{4}$ substrate.

(dotted lines in Fig. 9). This atomic arrangement model is the first step to understanding the mechanism of "pure" $a$-axis oriented YBCO thin film growth, and this model was clarified by using a $\{100\} \mathrm{Nd}_{2} \mathrm{CuO}_{4}$ substrate [17], Ce: $\mathrm{Nd}_{2} \mathrm{CuO}_{4}$ and $\mathrm{SrPrGaO}_{4}$, as described in Sec. 3 .

Just like the process of the original graphoepitaxial growth, solid-phase epitaxial (SPE) growth of the initial thin film (template) layer takes place so as to seed the layer on the surface corrugation during the heating of the pre-deposited film, as shown in Fig. 4. The atomic corrugation of the substrate surface is intrinsic in the $\mathrm{K}_{2} \mathrm{NiF}_{4}$ structure and fairly expected to act as effective nucleation sites for the initial growth stage of YBCO thin film. In fact, only the template-grown layer exhibited $c$-axis like X-ray diffraction pattern which may be of pseudo-cubic phase $[13,14]$, and $T_{\mathrm{c}-\text { zero }}$ was not observed down to $20 \mathrm{~K}$ with semiconductive behavior in resistance-temperature measurements. Therefore, we assume that SPE takes place so as to recrystallize disordered layer seeded on the atomic corrugation of substrate surface. Anyway, atomic-scaled surface corrugation of tetragonal $\mathrm{K}_{2} \mathrm{NiF}_{4}$ (100) substrates should act as nucleation sites for YBCO, during a successive increase in growth temperature, as illustrated in Fig. 4. Figure 10 shows an enlarged TEM image at the interface between $\mathrm{SrLaGaO}_{4}$ substrate and the "pure" $a$-axis YBCO thin film. Lattice planes seem to fairly continue with other distinct phases such as pseudo-cubic ones of YBCO, or distinct template-grown initial layer.

\section{Defects in "pure" $a$-axis YBCO thin films on (100) $\mathrm{SrLaGaO}_{4}$}

The RHEED patterns from the as-grown "pure" $a$-axis film were fairly spotty, which implies that film surface is atomically rough. Figure 11 shows a RHEED pattern observed along the $b$-axis direction of the film on $\mathrm{SrLaGaO}_{4}$. A spotty pattern proves that the surface is atomically rough. In fact, surface roughness was estimated to be roughly a few tens of nanometers from peak to valley. Figure 12 shows a low magnification of a cross-sectional TEM image at the interface viewed from the [010] direction of the substrate, on which it can be recognized that 
the substrate surface is somewhat wavy. Peak-to-valley distance was estimated to be $10-15 \AA$ and the peak-to-peak (or valley-to-valley) distance varied from about $250 \AA$ to more than $1000 \AA$. To confirm this interface irregularity, an as-polished substrate was heat-treated at $850^{\circ} \mathrm{C}$ for $30 \mathrm{~min}$ in vacuum, conditions similar to those prior to film deposition (see Fig. 4). Figure 13 shows an example of an AFM image of thus treated bare surface. Surface roughness was measured to be on the order start of of $10 \AA$. The surface exhibits hills which are roughly the same sizes

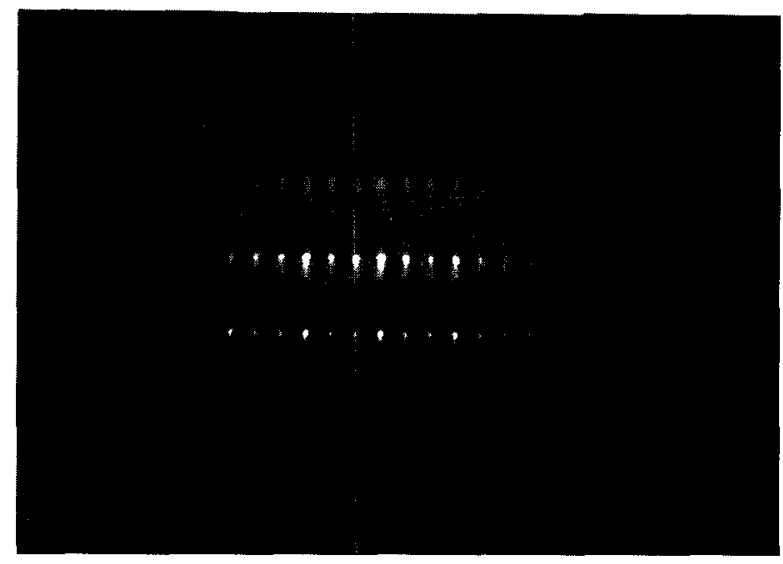

Fig. 11. Spotty RHEED pattern of the "pure" $a$-axis oriented YBCO thin film along the [010] direction.

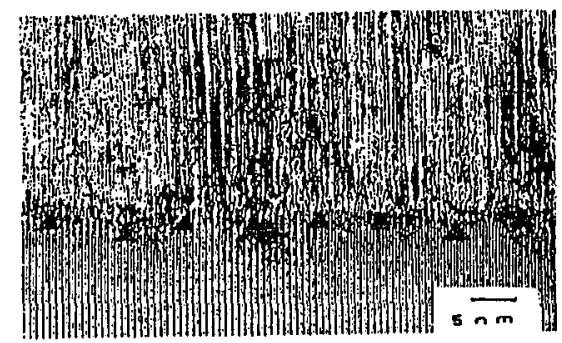

Fig. 12. Low magnification of TEM image at the interface, where substrate surface looks wavy as indicated by triangles.

as that estimated from the TEM picture shown in Figs. 10 and 12. Therefore, the interface roughness is due to the substrate surface itself, and causes surface roughness in the as-grown YBCO film.

This irregular interface feature also results in complicated microstructures in the film. Figure 14a shows a planar TEM image of the "pure" $a$-axis oriented film. Careful observations show that lattice planes look tangled, forming a kind of granular structure. Inter-lattice planes among adjacent regions connect alternately with a shift of roughly $c / 2$. This results in anti-phase domains, as schematically shown in Fig. 14b. The film has a granular structure with grains from 100 to $500 \AA$. 


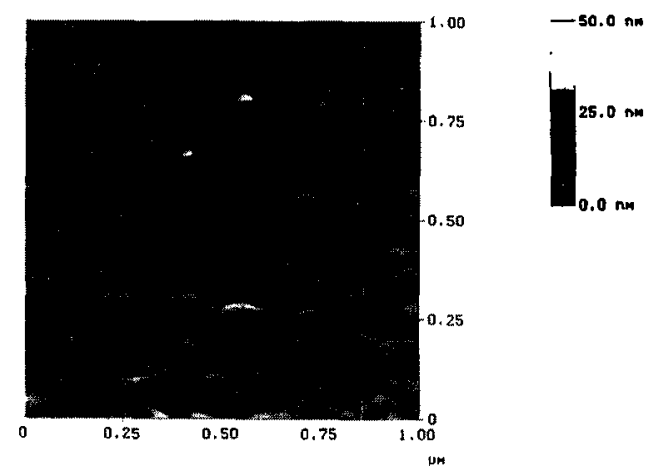

Fig. 13. AFM image of a heat-treated surface of (100) $\mathrm{SrLaGaO}_{4}$ substrate.
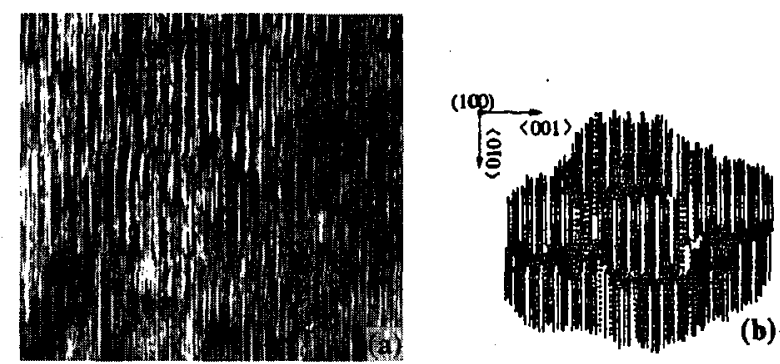

Fig. 14. Plane-view TEM image of the "pure" a-axis oriented YBCO film on $\mathrm{SrLaGaO}_{4}$ (a) and a schematic drawing of granular structure (b).
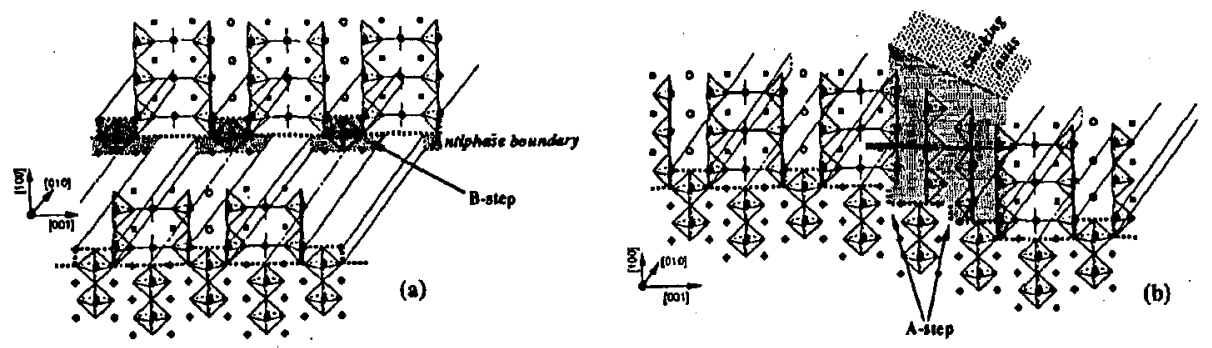

Fig. 15. Models for the formation of anti-phase boundary (a) and stacking-fault boundary (b). Steps $A$ and $B$ appear on a cobbled surface of $\mathrm{SrLaGaO}_{4}$ substrate.

This size is comparable to surface morphology of the substrate surface, as shown in Fig. 13.

To understand tangled microstructures viewed from both [010] and [100] directions, we re-examined the substrate surface. As shown in Fig. 13, when the (100) surface of $\mathrm{SrLaGaO}_{4}$ is annealed in oxygen atmosphere at $850^{\circ} \mathrm{C}$ for $30 \mathrm{~min}$, the surface morphology changes from an as-polished smooth surface to a cobbled surface. In the crystal structure of $\mathrm{K}_{2} \mathrm{NiF}_{4}$ material, the position of an oxygen 
octahedron sites at $(1 / 2,1 / 2)$ from $(0,0)$ along both $c$ - and $a$-axis directions. Figure 15 shows a schematic drawing of the tangled microstructure [20]. On part of the cobble along the [010] direction of the substrate, the surface grooves/ridges made of oxygen octahedra shift by one half of the $c$-axis length, by which a single step ( $B$-step) appears along the [010] direction, as shown in (a) by dotted lines. According to our atomic graphoepitaxial relationship between $\mathrm{YBCO}$ and $\mathrm{K}_{2} \mathrm{NiF}_{4}$ structure, $-\left\{\mathrm{CuO}_{2}-\mathrm{BaO}-\mathrm{CuO}-\mathrm{BaO}-\mathrm{CuO}_{2}\right\}-$ block and $-\left\{\mathrm{CuO}_{2}-\mathrm{YO}-\mathrm{CuO}_{2}\right\}-$ block in YBCO structure gear with the groove and ridge of the substrate surface, respectively, as shown in Fig. 9. Therefore, these blocks on $B$-steps shift by one half the $c$-axis length from those on the understep part. As a result, an anti-phase boundary forms at the step perpendicular to the [010] direction. On part of the cobble along the [001] direction of the substrate, on the other hand, surface grooves/ridges make steps ( $A$-step) of one half the $a$-axis length along the [001] direction, as shown schematically in (b). Therefore, a kind of stacking-fault like lattice defects is formed on these steps, which runs along the [010] direction. Relatively defect-free grains are surrounded by a combination of these inter-lattice defects.

At the initial growth stage of pure $a$-axis oriented YBCO thin film, surface steps along either the [010] or [001] directions must act as nucleation sites of YBCO embryos, resulting in defect boundaries made of stacking variations. Granular structure in the "pure" a-axis YBCO thin films on the (100) $\mathrm{SrLaGaO}_{4}$ consists of these boundaries. The as-polished (100) surface of $\mathrm{SrPrGaO}_{4}$ was more tough against the heat treatment, and the studies on microstructures in the "pure" $a$-axis films on it is now under going.

Why the (100) surface of $\mathrm{SrLaGaO}_{4}$ exhibits cobbled irregularity by pre-annealing at $850^{\circ} \mathrm{C}$ is not clear, but it can be speculated that the crystal grows with cellular structure, even if growth conditions are optimized, since the single crystal is usually pulled from an off-stoichiometric melt because of its incongruent melting [21]. Cellular structure in cross-section shows a kind of honeycomb made of ordered and disordered parts, that is, mosaic structure, which must be the origin of cobbled surface shown in Fig. 13. Therefore, high quality $\mathrm{SrLaGaO}_{4}$ single crystals without any sub-structures are strictly demanded in order to suppress anti-phase and stacking-fault boundaries in YBCO thin films.

\section{Summary}

The preferred orientation of $\mathrm{YBa}_{2} \mathrm{Cu}_{3} \mathrm{O}_{x}$ thin films grown on pseudo-cubic perovskite-related substrates was reviewed. It was clearly found that $a$-axis oriented grains in $c$-axis dominated films on (110) $\mathrm{NdGaO}_{3}$ substrates had $c$-axis in-plane alignment on tetragonal substrate surface. This finding moved us to use tetragonal $\mathrm{K}_{2} \mathrm{NiF}_{4}$-structure substrate materials for obtaining the "pure" $a$-axis oriented YBCO thin films. On (100) $\mathrm{SrLaGaO}_{4}, \mathrm{Nd}_{2} \mathrm{CuO}_{4}, \mathrm{Ce}: \mathrm{Nd}_{2} \mathrm{CuO} \mathrm{O}_{4}$ and newly developed $\mathrm{SrPrGaO}_{4}$ substrates, the "pure" a-axis oriented YBCO thin films were obtained successfully. The novel epitaxial mechanism called "atomic graphoepitaxy" was presented. However, anti-phase and stacking-fault boundaries existed in the film on the (100) $\mathrm{SrLaGaO}_{4}$ substrate. A model of anti-phase domains/boundaries formation in "pure" $a$-axis oriented $\mathrm{YBa}_{2} \mathrm{Cu}_{3} \mathrm{O}_{x}$ thin films was 
fairly well explained by atomic graphoepitaxial growth on cobbled surfaces inherent in (100) surface of $\mathrm{SrLaGaO}_{4}$. Other $\mathrm{K}_{2} \mathrm{NiF}_{4}$ compounds such as $\mathrm{SrPrGaO}_{4}$ are expected to be potential candidates.

\section{Acknowledgments}

The authors would like to express their thanks to Prof. H. Kojima and Dr. I. Tanaka of Yamanashi University for supplying Ce: $\mathrm{Nd}_{2} \mathrm{CuO}_{4}$ crystals, to Dr. H. Sato, NTT Basic Research Laboratories, for supplying $\mathrm{Nd}_{2} \mathrm{CuO}_{4}$ single crystals and to our colleagues Dr. Y. Tazoh and Dr. T. Itoh for their helpful comments.

\section{References}

[1] H.J. Scheel, MRS Bull. XIX, 26 (1994).

[2] S. Miyazawa, M. Mukaida, in: Advances in Superconductivity VIII, Eds. H. Hayakawa, Y. Enomoto, Springer-Verlag, Tokyo 1996, p. 949.

[3] Y. Tazoh, S. Miyazawa, Appl. Phys. Lett. 62, 408 (1993).

[4] For example, M. Hawley, I.D. Raistric, J.G. Beery, R.J. Houlton, Science 251, 1587 (1991).

[5] C.B. Eom, A.F. Marchall, S.S. Landerman, R.D. Jocowitz, T.H. Geball, Science 249, 1549 (1990).

[6] M. Mukaida, S. Miyazawa, J. Appl. Phys. 74, 1209 (1993).

[7] J. Kobayashi, M. Mukaida, Y. Tazoh, S. Miyazawa, in: Advances in Superconductivity VII, Eds. K. Yamafuji, T. Morishita, Springer-Verlag, Tokyo 1995, p. 905.

[8] M. Mukaida, S. Miyazawa, Jpn. J. Appl. Phys. 32, 4521 (1993).

[9] M. Sasaura, S. Miyazawa, M. Mukaida, J. Appl. Phys. 68, 3643 (1990).

[10] M. Mukaida, S. Miyazawa, Jpn. J. Appl. Phys. 31, 3317 (1992).

[11] S. Hontsu, N. Mukai, T. Kawai, S. Kawai, Appl. Phys. Lett. 61, 1134 (1992).

[12] M. Mukaida, S. Miyazawa, Appl. Phys. Lett. 63, 999 (1993).

[13] J.A. Agostinelli, S. Chen, G. Braunstein, Phys. Rev. B 43, 11396 (1991).

[14] J.G. Wen, S. Mahajan, W. Ito, T. Morishita, N. Koshizuka, Appl. Phys. Lett. 64, 3334 (1994).

[15] M. Mukaida, Phys. Rev. B 50, 7124 (1994).

[16] M. Sasaura, S. Miyazawa, J. Cryst. Growth 166, 825 (1996).

[17] S. Miyazawa, M. Mukaida, Appl. Phys. Lett. 64, 2160 (1994).

[18] D.C. Flanders, H.I. Smith, J. Vac. Sci. Technol. 15, 1001 (1978).

[19] H.I. Smith, private communication, 1994.

[20] S. Miyazawa, M. Mukaida, Jpn. J. Appl. Phys. 35, L1177 (1996).

[21] A. Dabkowski, H.A. Dabkowska, J.E. Greedan, J. Cryst. Growth 132, 205 (1993). 